\title{
Rehabilitation Outcomes of Persons with Complete Paraplegia at a Private Rehabilitation Hospital
}

\begin{abstract}
In order to overcome activity limitations, prevent secondary complications and early death and achieve community integration comprehensive rehabilitation post spinal cord injury (SCI), is essential. The aim of the study was to evaluate outcomes of patients with complete paraplegia who received rehabilitation at a private rehabilitation hospital.

A quantitative, descriptive methodology was implemented. All patients with complete, traumatic, thoracic spinal cord injuries, admitted to the study hospital in the study period, were consecutively sampled. Thirty five patients were identified of whom 16 adhered to the inclusion criteria. The Functional Independence Measure (FIM) and Needs Assessment Checklist (NAC) were used as measuring instruments.

The mean length of stay was 95 days. Discharge FIM motor scores ranged from 72 to 83 with a mean of 79.3. The mean gain in FIM motor score was 55 and varied from 45 to 61. Discharge NAC scores ranged from 264 to 340 with a mean of 300 out of a possible 347. A correlation between length of stay and discharge FIM scores $(p=0.05)$ were found.

Both NAC and FIM scores indicated high levels of physical independence. According to NAC scores patients were educated on the prevention of secondary complications and received psychological counselling. Discharge planning and community integration scored lower with means of $80 \%$ and $61 \%$ respectively. Community based completion of rehabilitation programmes, the incorporation of the NAC, or another participation outcome measure and implementation of on-going programme monitoring and assessment strategies is recommended.
\end{abstract}

KEY WORDS: SPINAL CORD INJURIES, REHABILITATION PROGRAMME OUTCOMES, COMMUNITY INTEGRATION.

\section{INTRODUCTION}

Spinal Cord Injury (SCI) is a relatively uncommon yet devastating cause of disability (McKinley et al, 2007). Persons with spinal cord lesions are confronted with motor and sensory impairments as well as bladder and bowel dysfunction, which cause activity limitations and can have a severe impact on participation in life (McKinley et al, 2007). The nature and severity of activity limitations and participation restrictions are dependent on the severity and site of the lesion as well as the person's social roles and contextual factors (McKinley et al, 2007). Numerous secondary complications may arise from SCI (Eng \& Miller 2008). Thus comprehensive rehabilitation post spinal cord injury is crucial to prevent complications such as pressure ulcers and early mortality (Krause et al, 2008), to improve function and to assist with community reintegration and economic self sufficiency (Schönherr et al 1999).
Rehabilitation for SCI individuals is highly specialised and traditionally occurs in institutions such as tertiary hospitals and rehabilitation centers (Schönherr et al 1999). The Consortium for Spinal Cord Medicine (1999), have published clinical guidelines re expected functional outcomes following traumatic spinal cord injury. Functional recovery can be measured by various outcome measures including the Modified Bartel Index (MBI), the Quadriplegia index of function (QIF), the Functional Independence Measure (FIM) and the Spinal cord independence measure (SCOM) (Anderson et al, 2008) to name but a few. The above measures are objective tools that do not take into consideration the patient's opinion and needs. In addition they measure activity and do not include participation measures such as community integration and employment. However, it is clear from the International Classification of Function,
Disability and Health's (ICF) definition that rehabilitation should encompass all measures necessary to maximize physical and psychological health, including social, economic and vocational aspects and that the person and his/her opinion plays an important role in determining outcomes (WHO 2000). Therefore subjective tools that take the patient's opinion into account and participation measures must be included in outcome measurement studies and practices.

The Rehabilitation Hospital, where this study was conducted, is a 120 bed private rehabilitation hospital in

Corresponding Author:

Rita Henn

P.O. Box 9235

Centurion

0046

South Africa.

E-mail: rhenn@worldonline.co.za 
Gauteng, where a rehabilitation program for patients with paraplegia, amongst others, is being offered by an interdisciplinary rehabilitation team. The overall aim of the rehabilitation program for patients with paraplegia at the rehabilitation hospital is to ensure that the patient achieves physical independence using a wheelchair and to prepare the patient for integration back into the community. The outcomes of the program have not been assessed since its inception in 1999. It was therefore unknown whether the program achieved its aims as stated above. Thus the current study evolved to assess the outcomes of patients with complete paraplegia who received rehabilitation at this hospital. Specific objectives included determining whether there were any relationship between length of stay and FIM and/or Needs Assessment Checklist (NAC) scores, how functional outcomes of patients according to FIM and NAC scores compared to expected outcomes according to the Clinical Practice Guidelines (CPG) of the Consortium for Spinal Cord Medicine (1999) and assess community integration outcomes of patients according to NAC scores.

\section{METHODOLOGY}

The study was registered with the University of Stellenbosch's Committee for Human Research. Permission to perform the study and access patient records was obtained from the director of the hospital. Participation in the study was voluntary and every study participant signed a consent form before participating in the study. Information disclosed by participants was kept confidential.

A quantitative, descriptive methodology was implemented. The study population consisted of a consecutive sample of all patients admitted to the study hospital with a complete, traumatic, thoracic spinal cord injury, classified as ASIA A, by a the medical doctor at the study hospital, during the period; 1 April 2004 to 31 December 2007. A total of 35 patients were identified. Of these, 19 had to be excluded according to the study's exclusion criteria; eight suffered from systemic conditions such as cardiovascular disease, which might impact negatively on outcomes, nine were discharged before completion of rehabilitation in the opinion of the rehabilitation team and two died during rehabilitation, thus their individual outcomes could not be assessed. Thus 16 patients participated in the study.

The FIM and Needs Assessment Checklist (NAC) were used as measuring instruments in the study. The FIM, a valid, reliable and sensitive instrument, was chosen primarily since it is the only outcome measure currently in use in the study setting. It measures the functional independence of a person on 18 activities that are each scored on a seven point scale $(1-7)$ (Masedo, et al 2004). For the purposes of this study only the motor subscale of the FIM were used as suggested by Anderson et al (2008): "If the FIM were to be used in clinical research settings to assess motor function, it may be more useful to only use the motor subscale portion of the FIM and disregard the cognitive subscale portion". The study hospital is subscribed to use the FIM, a copyrighted instrument, and pays a monthly license fee, which includes using it for research purposes. The primary author of this article completed all the FIM assessments. She is internationally certified to use the FIM and has been using it regularly for eight years. In accordance with FIM guidelines admission scores were determined within three days after admission (Anderson et al 2008). Discharge FIM scores were determined by the same person one day before discharge. Both assessments were completed in the spinal ward of the hospital using the patient's hospital bed and the ward's bathroom. The curtains were drawn around the bed to ensure patient privacy and to prevent interruptions.

The Needs Assessment Checklist it is a valid, reliable and sensitive tool that can assist with focussing rehabilitation programs on individual patients needs (Berry \& Kennedy 2002). The checklist covers nine domains: activities of daily living, skin management, bladder management, bowel management, mobility, equipment, community preparation, discharge coordination and psychological issues (Kennedy et al 2003).

Table 1: Demographic profile and information on injury

\begin{tabular}{|c|c|c|}
\hline Gender distribution & Male & 13 \\
\hline & Female & 3 \\
\hline \multirow[t]{3}{*}{ Age distribution } & $21-20$ & 7 \\
\hline & $31-40$ & 7 \\
\hline & $41-50$ & 2 \\
\hline \multirow[t]{3}{*}{ Educational level } & Grade 8 & 5 \\
\hline & Grade 10 & 5 \\
\hline & Tertiary education & 6 \\
\hline \multirow[t]{2}{*}{ Pre injury employment status } & Employed & 15 \\
\hline & Student & 1 \\
\hline \multirow[t]{2}{*}{ Transport } & Own & 11 \\
\hline & Public & 5 \\
\hline \multirow[t]{6}{*}{ Level of injury } & T4 & 1 \\
\hline & T5 & 3 \\
\hline & T6 & 2 \\
\hline & T8 & 2 \\
\hline & T11 & 5 \\
\hline & T12 & 3 \\
\hline \multirow[t]{4}{*}{ Cause of injury } & Motor vehicle accident & 8 \\
\hline & Violence & 3 \\
\hline & Industrial & 4 \\
\hline & Fall & 1 \\
\hline
\end{tabular}


It is completed by the patient, who rates his/her level of independence for each item on a scale from 0 to 3 . No distinction is made between verbal and physical independence (Kennedy \& Hamilton 1999). The NAC was chosen as an additional measuring instrument in the study since it provides the patients' views of their functional ability and, in addition, assess issues related to the prevention of secondary complications, employment and community integration.

Participants completed the NAC on the day before discharge at a time convenient to them in a private, quiet room. The checklist and the scoring were explained individually to each participant by the primary author and they were allowed to fill it in with no time constraints. Explanations and translations were provided to non-English speaking participants through an interpreter. Not translating this instrument created bias as explanations from the translator might have changed between participants. The lack of an admission NAC score was a further shortcoming in the study.

All data was entered onto an excel spreadsheet and analysed with the assistance of a statistician using STATISTICA Version 7. Specific tests (Spearman; Kruskall Wallis) were done to determine statistical significance of selected findings such as the relationship between length of stay (LOS) and mean FIM admission and FIM and NAC discharge scores. A $p$ value of $<0.05$ was deemed statistically significant.

\section{RESULTS}

The demographic details of the study population are presented in table $\mathbf{1}$. The mean age of the study population was 32 years with ages ranging from 21 to 47.

All sixteen patients were from urban areas and resided in houses. All participants, except one who was a student, were employed and six had higher education. Motor vehicle accidents were the most common cause of injury.

The combined LOS in acute and rehabilitation hospitals ranges from 83 to 152 days with a mean of 113 days. The mean length of stay (LOS) in the rehabilitation hospital was 95 days (SD $=20.83$ ) with the length of stay ranging

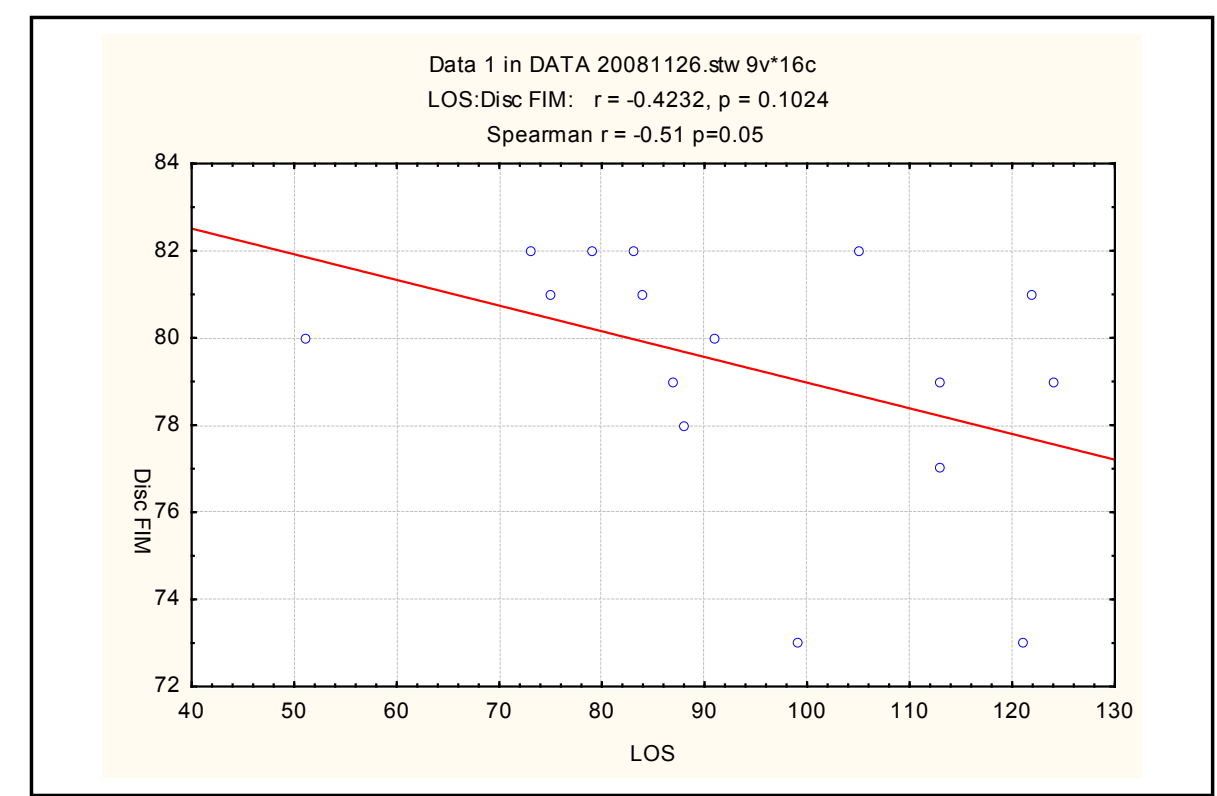

Figure 1: Length of stay compared to discharge FIM score

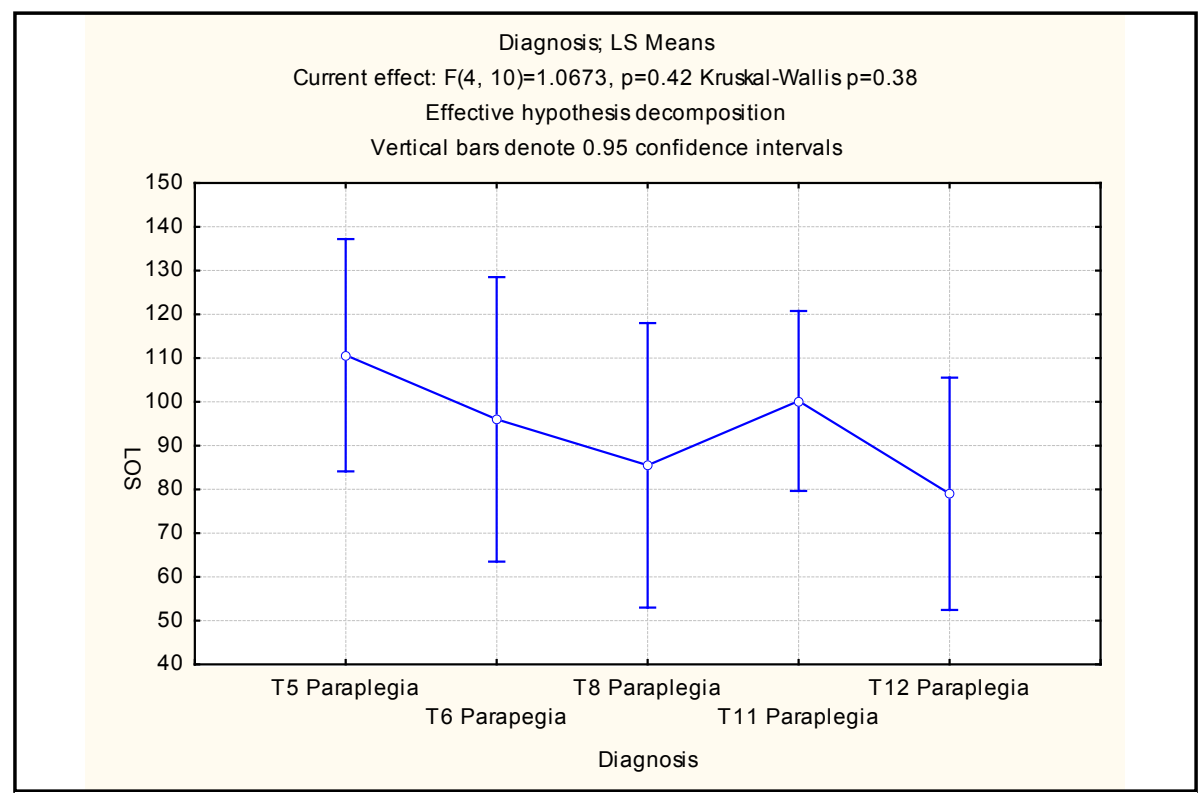

Figure 2: Level of lesion compared to length of stay

from 51 days to 124 days. Ten patients stayed longer than the expected 84 days as set out by the rehabilitation program. There was no correlation between length of stay and NAC $(\mathrm{p}=0.26 ; \mathrm{r}=-0.3)$ scores. According to figure 1 there was some correlation between length of stay and discharge FIM scores $(\mathrm{p}=0.05$, $\mathrm{r}=-0.51)$.

No statistically significant relationship could be found between LOS and the level of the lesion $(\mathrm{p}=0.38)$. However, clinically mean LOS decreased by 30 days from 110 days for T5 lesions to 80 days for T12 lesions as indicated in figure 2 .
The admission FIM motor score ranged from 23 to 27 with a mean of 24.3 $(\mathrm{SD}=1.19)$ out of a possible 91 . In the domains of bladder and bowel as well as transfers and mobility, areas where a patient with paraplegia has to acquire new skills, all patients scored the lowest possible score, i.e. one out of seven. Neither gender $(p=0.35)$ nor level of injury $(\mathrm{p}=0.39)$ or cause of injury $(p=0.83)$ had any significant impact on FIM admission scores.

Discharge FIM scores ranged from 72 to 83 out of a possible 91 . The mean FIM discharge motor score was 79.3 $(\mathrm{SD}=2.89)$. Most categories in the FIM showed gains in the mean score with 
the biggest improvements in the bladder and bowel and, the transfers and mobility categories. No relationship could be found between gender $(p=0.4)$, cause of injury $(\mathrm{p}=0.13)$ and level of injury $(\mathrm{p}=0.65)$ (figure 3) and FIM discharge scores.

The mean gain in FIM motor score was 55 and varied from a low of 45 to a high of 61 . There was no statistically or clinically significant relationship between admission and discharge FIM scores.
NAC scores were determined only on discharge and ranged from 264 to 340 with a mean of $300(\mathrm{SD}=22.22)$ out of a possible 347 . No significance could be found between gender $(p=0.19)$, level of lesion $(\mathrm{p}=0.67)$ and cause of injury $(\mathrm{p}=0.95)$ and NAC scores.

Findings from the FIM and NAC were translated into percentages in order to compare them to each other and the Consortium for Spinal Cord Medicine (1999) guidelines. The FIM and NAC

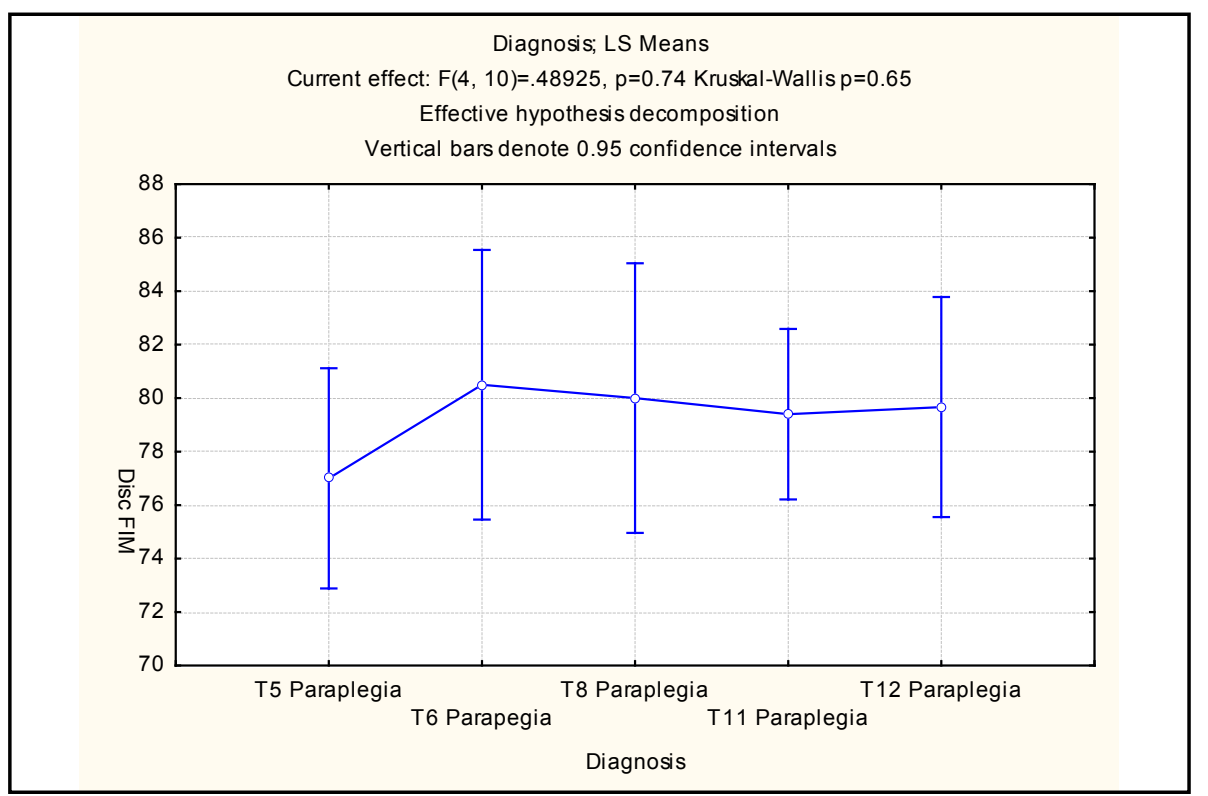

comparative findings are presented in Table 2.

With regards to self-care scores include the motor FIM scores in the Self-care category i.e. eating, grooming, bathing, toileting and dressing upper and lower body. NAC scores include scores in the Activities of Daily Living category i.e. food management, facial hygiene, personal hygiene and dressing upper and lower body. The mean percentage for the FIM self-care score was 95 . $5 \%$, with a median of $97 \%$ while the NAC mean percentage was $99 \%$ and the median $100 \%$. It is evident that the patients' perception of their independence in self-care is better than what was reflected by objective FIM scores.

The lower mean score in bladder and bowel management achieved in the FIM can possibly be explained by the fact that patients with paraplegia are taught to empty their bladders with the aid of a catheter in the rehabilitation unit where the study was performed. Thus no paraplegic could score the maximum of seven for this category even if completely independent in this activity.

For the mobility FIM scores, the patients were observed while doing transfers in hospital to their beds, the toilet, a bath and a car. They were also observed

Figure 3: FIM discharge scores compared to level of injury

Table 2: Comparison of FIM discharge and NAC motor scores in percentages

\begin{tabular}{|c|c|c|c|c|c|c|c|}
\hline Variable & Mean & SD & Median & Minimum & Maximum & $\begin{array}{l}\text { Lower } \\
\text { quartile }\end{array}$ & Upper quartile \\
\hline FIM - Self care & 95.5 & 3.52 & 97.0 & 90 & 100 & 93.00 & 97.00 \\
\hline NAC - Self care & 98.94 & 2.02 & 100.00 & 94 & 100 & 99 & 100 \\
\hline FIM - Bladder \& Bowel & 85.56 & 1.75 & 86 & 79 & 86 & 86 & 86 \\
\hline NAC - Bladder \& Bowel & 91.06 & 13.81 & 97 & 51 & 100 & 91 & 100 \\
\hline FIM - Mobility & 76.19 & 4.69 & 77 & 66 & 80 & 74 & 80 \\
\hline NAC - Mobility & 95.13 & 5.57 & 97 & 82 & 100 & 92.5 & 100 \\
\hline
\end{tabular}

Table 3: NAC scores in domains related to discharge and community integration

\begin{tabular}{|l|l|l|l|l|l|l|l|}
\hline Variable & Mean & SD & Median & Minimum & Maximum & $\begin{array}{l}\text { Lower } \\
\text { quartile }\end{array}$ & $\begin{array}{l}\text { Upper } \\
\text { quartile }\end{array}$ \\
\hline Wheelchair & 85.69 & 21.78 & 98 & 33 & 100 & 74.5 & 100 \\
\hline Community integration & 80.19 & 14.64 & 84 & 55 & 100 & 64.5 & 90.5 \\
\hline Discharge planning & 61.25 & 20.83 & 56 & 35 & 100 & 44 & 73.5 \\
\hline Psychological counselling & 88.38 & 9.97 & 91 & 73 & 100 & 79 & 97 \\
\hline Prevention of complications & 95.93 & NA & 100 & 85 & 100 & 88 & 100 \\
\hline
\end{tabular}


while propelling a wheelchair on a level surface for $50 \mathrm{~m}$. On comparing the FIM and NAC scores it is again noted that the NAC mean (95) is higher than that of the FIM (76). No patient was scored more than $80 \%$ independent in transfers and mobility, while no patient scored him/ herself lower than $80 \%$ independent in the tasks that were part of this category.

The expected FIM outcome according to the Consortium for Spinal Cord Medicine (1999) is $86-100 \%$ for selfcare activities, bowel management and transfers, while it is $86 \%$ for bladder management and wheelchair mobility. Thus the results of this study correlated well with the expected outcomes for self-care, bladder and bowel management. The lower mobility FIM scores (66 to $80 \%$ ) could be because the patients were scored on four different transfers while the CPG only refers to bed-wheelchair transfer.

Scores from additional NAC categories as summarised in Table 3 indicated that prevention of secondary complications had been addressed to the patients' satisfaction by the rehabilitation programme.

Scores for knowledge on and maintenance of wheelchair and equipment, were good but a low of 33 indicates that this is an area that requires attention. Similarly scores for psychological counselling indicates that the programme fared well in this respect. The two outcomes that raised concern about the program were discharge planning and community integration. Community integration includes social activities, employment, community preparation and, driving and transport issues. On closer scrutiny it was found that the questions about employment were scored the lowest. Seven patients reported that adaptations were necessary for them to return to work and that at the time of discharge no adaptations were made, three patients also reported that they had made no plans to return to work. No work visits were done by the rehabilitation team.

The discharge planning category includes community issues, accommodation and carer training. The questions focus on the patient's preparation for discharge, adaptations to their houses and on knowledge regarding where to find medical assistance in their communities. In this area twelve of the patients scored lower than $70 \%$ with six scoring below $50 \%$ and four above $80 \%$. Six patients did not receive a home visit to assist them with the planning of home adaptations and 11 (69\%) did not know where to access specialised medical support in the community after discharge.

\section{DISCUSSION}

While the demographic details of the study population such as gender ratio (4:1) and mean age at the time of the injury (32) correlates with literature (Blackwell et al, 2001; Wyndaele \& Wyndaele 2006; McKinley et al 2007; Wirth et al 2010), the authors want to caution readers to carefully consider the demographic details and study-setting for similarities and differences before extrapolating results since the study population consisted of a small, homogenous group, with no systemic diseases, from a privileged setting who could afford medical insurance and private rehabilitation.

The patient's physical outcomes were good. Both objective scores from the FIM as well as subjective NAC scores indicated positive outcomes in this area and it was confirmed by the favourable comparison of FIM scores with the expected scores from the CPG (1999).

The length of stay for paraplegics undergoing rehabilitation at the study hospital is set at 84 days. When one reviewed the LOS figures from Schönherr et al 1999 (mean $=157$ days $)$ and Wirth et al (2010) (mean $=250$ days) it is not surprising that ten participants in the current study stayed longer than the preordained 84 days. The need for a rehabilitation period longer than 84 days, whether in patient or community based is supported by a study which measured physical progress post SCI, with the SCIM (Wirth et al, 2010). Wirth et al (2010) found a mean improvement of 39 points after 4 months (120 days) and a further mean improvement of 11 points after three more months (90 days or 210 days in total).

When one takes into consideration that nine patients were discharged home early, in the opinion of treating professionals, from the programme under study, because of a lack of funds, it appears that length of stay at the study hospital is to a large extent determined by the insurance companies' payment policies rather than by the patients' needs. This indicates a need to educate insurers about the rehabilitation needs of SCI patients' and the need to explore the option of community based rehabilitation programs if the cost of hospitalisation proves to be an inhibiting factor for optimal rehabilitation. Another possible solution could be that the rehabilitation program for SCI patients be changed, in accordance with The National Rehabilitation Policy of South Africa (Department of Health, 2000), to incorporate an institution based phase and a community based phase to ensure patients receive the necessary length of rehabilitation required.

Patients showed marked functional improvement as measured by the FIM and NAC. Thus the rehabilitation program is effective in terms of addressing patient's needs for physical independence. While these findings reflected well on the rehabilitation program, one must raise the question of why the FIM is used as the only outcomes measure in the programme since it is according to a literature review on the subject "not a suitable outcome tool" to "detect small but functionally significant amounts of change in the SCI population" (Anderson et al, 2008 p.141). The challenges with regard to responsiveness experienced when using the FIM in the SCI population was illustrated in this study again. The NAC score range in bladder and bowel management (51 to 100) was much wider than that of the FIM (79 to 86) as well as a ceiling effect and underscoring in sphincter management and mobility since the use of assistive devices makes it impossible to have a perfect score on the FIM. However, the patients saw themselves as independent in these areas as the perfect NAC scores indicated. Thus even as an activity measure the FIM showed shortcomings for this type of population and the SCIM III (Anderson et al, 2008) would be a more suitable alternative, while a participation measure such as the NAC or participation subset of the International Classification of Function (ICF) (WHO, 2000) should also be implemented. 
To properly asses the outcomes of a rehabilitation program one must include participation measures to assess; community integration, employment and preparation for these during rehabilitation. The majority of the patients in this study were of an age to return to meaningful employment after rehabilitation. However, the NAC results indicated that according to patient perception the program experienced challenges in this regard, for example no work visits were undertaken by the rehabilitation team in the current study. This lack in SCI rehabilitation services was also identified in other studies as presented by Ottomanelli et al (2009) in a review on the literature of employment of persons with spinal cord injuries. It is essential that the rehabilitation programme focus on assisting persons with a disability to return to employment.

The rehabilitation programme must be extended to provide support on issues like reasonable accommodation, especially since South African legislation supports this process through the Employment Equity Act (RSA, 1998), the Code of Good Practice (RSA, 2002) and the Technical Assistance Guidelines (Department of labour, 2002). The study participants had some advantages over the general population for to returning to employment such as being of a young age, having a higher qualification in $38 \%$ of cases and having been employed in administrative work before the injury (Ottomanelli et al 2009). Higher levels of education provide a person with increased choice in type of employment, often require less change in type of employment and usually indicate individuals with more autonomy and motivation (Ottomanelli et al 2009). One of the main barriers to employment after spinal cord injury is dependency on public transport (Ottomanelli et al, 2009). Therefore, the five participants in the current study who are dependent on public transport might have more problems than their counterparts to secure and maintain employment, especially due to the lack of accessible public transport in SA (Howell et al 2006).

Another area which showed poorer outcomes was the referral of patients to community health care services.
Since the study hospital does not follow up patients, it is therefore, essential that patients are referred to community based service providers knowledgeable about the condition of SCI and its management, especially the prevention and management of secondary complications. Persons with SCI are prone to a myriad of secondary complications such as, bladder infections, pressure sores, respiratory infections and depression that can lead to high levels or morbidity and early mortality (Krause et al, 2008). Education about the maintenance of adequate health should be an essential part of a rehabilitation program (Ottomanelli et al, 2009). This was addressed in part by the current programme, since the NAC scores indicated high levels of education on prevention of complications, but the program failed to supply patients with knowledge about where to go for treatment of these problems should they occur.

\section{CONCLUSION}

The overall aim of the rehabilitation program at the study hospital is to prepare the patient for functional independence in a wheelchair and to successfully integrate back to his/her community. This study found that the patient was functionally independent in physical activities by the time of discharge, but that the rehabilitation program was not equally effective in the preparation for integration into the community. While there were some attempts to prepare the patient for participation and social roles, the bulk of the program is activity focused, and did not assist the patients after discharge from the hospital with the actual community reintegration process.

\section{RECOMMENDATIONS}

A rehabilitation program which combines a shorter in-patient phase to address issues like bladder and bowel management, and basic self-care, with a community based phase that completes these aspects of rehabilitation and also addresses home, community and work integration is recommended. The development of such programs will need careful consideration, planning and research. The South African Spinal Cord Association could play an inva- luable role with regard to developing and initiating such programs.

With regards to outcome measures used the results of the study had shown a need for an activity measure more specifically focused on individuals with spinal cord injuries such as the SCIM III or the NAC. The NAC has been found to be a suitable instrument to measure both physical independence and the extent to which patient needs had been met in terms of preparation for participation in social roles. Therefore it is recommended that the NAC is introduced as a measuring instrument into the spinal cord injury rehabilitation program at the study hospital. There is a need to complete the NAC during the rehabilitation process and on discharge. Since the rehabilitation period is set arbitrarily at twelve weeks and the issues that were found lacking in the rehabilitation program according to the NAC focused mainly on community preparation and discharge coordination, areas that are traditionally addressed during the more advanced stages of rehabilitation, it is recommended that the NAC is initially completed after the first month of rehabilitation. This information can then be used to set specific patient-centred goals with regards to community integration for the final two months of rehabilitation.

It is further recommended that performance measurement becomes an integral part of the rehabilitation program at the study hospital and is performed on a regular basis. This will provide management with feedback on programme efficiency, quality and effectiveness that can be implemented to continuously improve on the programme.

\section{DECLARATION OF CONFLICT OF INTEREST}

While not being employed by the study hospital the primary author and researcher does work in the facility. She did not treat any of the study participants. 


\section{REFERENCES}

Anderson, K. Aito, S. Atkins, M. BieringSørensen, F. Charlifue, S. Curt, A. Ditunno, J. Glass, C. Marino, R. Marshall, R. Mulcahey, M.J. Post, M. Savic, G. Scivoletto, G and Catz, A 2008 Functional Recovery Measures for Spinal Cord Injury: An Evidence-Based Review for Clinical Practice and Research J Spinal Cord Med. 31(2): 133-144.

Berry C \& Kennedy P 2002 'A Psychometric analysis of the Needs Assessment Checklist (NAC)'. Spinal Cord 41, 490-501.

Blackwell T, Krause J, Winkler T \& Stiens S 2001 Spinal Cord Injury Desk Reference: Guidelines for Life care planning and Case management. Demos Medical Publishing Inc. New York. P133-137.

Consortium for Spinal Cord Medicine 1999, Outcomes following traumatic spinal cord injury: Clinical Practice Guidelines for Health Care Professionals. Paralysed Veterans of America.

Department of Health 2000 National Rehabilitation Policy. Rehabilitation for all.

Department of Labor 2002 Technical Assistance Guidelines on the Employment of People with Disabilities (TAG). Pretoria: Department of Labor.

Eng J and Miller W 2008 'Rehabilitation: From bedside to community following spinal cord injury (SCI)'. Spinal Cord Injury Rehabilitation Evidence Version 2.0. Vancouver. p1.1-1.11.

Howell, C. Chalken, S. and Alberts, T 2006 "A history of the disability rights movement in South Africa" In Watermeyer, B. Swartz, L. Lorenzo, T. Schneider, M. and Priestley, M. (Eds.). Disability and social change: A South African Agenda 1st Ed. Cape Town: Human Sciences Research Council.

Kennedy P \& Hamilton L 1999 'The needs assessment checklist: a clinical approach to measuring outcome'. Spinal Cord 37, 136-139.

Kennedy P, Evans M, Berry C \& Mullin J 2003 'Comparative analysis of goal achievement during rehabilitation for older and younger adults with spinal cord injury'. Spinal Cord 41, 44-52.

Krause, J.S. Carter, R. Pickelsimer, E.E and Wilson D 2008 A prospective study of health and risk of mortality after spinal cord injury Arch Phys Med Rehabil. 89(8): 1482-1491.

Masedo AI, Hanley M, Jensen MP, Ehde D \& Cardenas DD 2004 'Reliability and validity of a self-report FIM ${ }^{\mathrm{TM}}$ (FIM-SR) in persons with amputation or spinal cord injury and chronic pain'. Am J Phys Med Rehabil 84, 167-176.
McKinley W, Santos K, Meade M \& Brooke K 2007 'Incidence and outcomes of spinal cord injury clinical syndromes'. Journal of Spinal Cord Medicine Vol 30 No 3 215-224.

Ottomanelli, L and Lind L 2009 Review of Critical Factors Related to Employment After Spinal Cord Injury: Implications for Research and Vocational Services J Spinal Cord Med. 32(5): 503-531

Republic of South Africa,(RSA) 2002 Employment Equity Act 5/1998: Code of Good Practice. Key Aspects of Employment of People with Disabilities. Government Gazette , 446 (23702).

Republic of South Africa 1998 Employment Equity Act No.55 :1998 http://www.dpsa.org. za/policies/employmentequityact.php Retrieved 04.2010

Schönherr M, Groothoff J, Mulder G \& Eisma W 1999 'Functional outcome of patients with spinal cord injury: rehabilitation outcome study'. Clinical Rehabilitation 13, 457-463.

Wirth, B. van Hedel, H.J.A. Kometer, B. Dietz V and Curt A 2008 Changes in Activity After a Complete Spinal Cord Injury as Measured by the Spinal Cord Independence Measure II Neurorehabil Neural Repair 22(2) 145 - 153

World Health Organization 2000 International Classification of Functioning (ICF).

Wyndaele M, Wyndaele J-J 2006 "Incidence, prevalence and epidemiology of spinal cord injury: what learns a worldwide literature survey?' Spinal Cord 44, 503-508. 